\title{
ON COLLECTIONWISE NORMALITY OF LOCALLY COMPACT, NORMAL SPACES
}

\author{
ZOLTÁN BALOGH
}

\begin{abstract}
We prove that by adjoining supercompact many Cohen or random reals to a model of ZFC set theory, in the resulting model, every normal locally compact space is collectionwise normal. In the same models, countably paracompact, locally compact $T_{3}$-spaces are expandable. Local compactness in the above theorems can be weakened to being of point-countable type, a condition that is implied by both Cech-completeness and first countability.
\end{abstract}

\section{INTRODUCTION}

This paper is devoted to the problem of deciding whether the following hypothesis and its countably paracompact analogue are consistent with the usual axioms of ZFC set theory.

The normal locally compact space hypothesis (NLCH). Every normal locally compact space is collectionwise normal.

Consistency is the best we can hope for, because in many models of ZFC the hypothesis is known to fail (see below). Historically, NLCH is part of the wider problem of when normal spaces are collectionwise normal and is a descendant of the normal Moore space problem that asks whether normal Moore (or, more generally, normal first countable) spaces are consistently collectionwise normal. The normal Moore space problem has induced an extremely strong activity from a large number of set-theoretic topologists (and set theorists) including many of the foremost contributors in the field (see the surveys $\left[T_{3}, F_{5}\right]$ ). After many partial answers, the final answer turned out to be this. If strongly compact or supercompact many random reals are added, by forcing, to a model of ZFC, then the Product Measure Extensions Axiom (PMEA) holds (K. Kunen; see $\left[\mathrm{F}_{5}\right]$ ). By an unexpected and elegant result of Nyikos, PMEA implies that normal first countable spaces are collectionwise normal $\left[\mathrm{N}_{1}, \mathrm{~N}_{2}\right]$. On the other hand, Fleissner $\left[\mathrm{F}_{3}\right]$ proved that if normal first countable spaces are collectionwise normal, then there are inner models with measurable cardinals. Thus the assumption of the consistency of large cardinals is indeed necessary.

Received by the editors November 15, 1987 and, in revised form, January 20, 1989.

1980 Mathematics Subject Classification (1985 Revision). Primary 54D15, 03E35; Secondary 54D45, $03 \mathrm{E} 55$.

Key words and phrases. Random and Cohen reals, forcing, supercompact cardinals, locally compact, collectionwise normal, normal Moore space conjecture, countably paracompact. 
The Kunen-Nyikos-Fleissner solution stimulated Tall, another main contributor to the topic, to develop direct methods of attacking the normal-impliescollectionwise-normal problem and to look for a significant context where this approach could be applicable. At about the same time, making use of $\left[\mathrm{F}_{1}\right]$, Watson $\left[\mathrm{W}_{1}\right]$ proved the nice result that under $V=L$ normal locally compact spaces were collectionwise normal with respect to compact subsets. He was the first to ask whether NLCH was consistent, although, as will turn out from the lists below, special cases of the problem had long been worked on before. Watson's problem made Tall $\left[\mathrm{T}_{8}\right]$ put forward the

Toronto project. Prove the consistency of $\mathrm{NLCH}$ from the consistency of ZFC with appropriately large cardinals.

Making progress, Tall $\left[\mathrm{T}_{2}-\mathrm{T}_{9}\right]$ actually carried out the Toronto project for spaces of character $\leq \beth_{\omega}$ (see (15) below). Other researchers also made significant contributions to the theme. A survey of these will be condensed in the following two lists of results, (1)-(15) and (a)-(e).

Locally compact normal spaces are (consistently) collectionwise normal if they are

(1) metacompact and perfect (in $\mathrm{ZFC},\left[\mathrm{A}_{2}\right]$ );

(2) locally connected and Moore (in ZFC, [RZ, CZ, AZ]);

(3) locally connected and submetacompact (in $\mathrm{ZFC},\left[\mathrm{G}_{1}\right]$ );

(4) boundedly metacompact (in ZFC, $\left[D_{1}\right]$ );

(5) screenable (in $\mathrm{ZFC},\left[\mathrm{B}_{1}\right]$ );

(6) connected and submetalindelöf $\left(2^{\omega_{1}}<2^{\omega}\right.$, [ $\left.\left.\mathrm{B}_{1}\right]\right)$;

(7) locally connected and perfect $\left(M A\left(\omega_{1}\right),[\mathrm{R}, \mathrm{L}]\right)$;

(8) Moore $\left(V=L,\left[\mathrm{~F}_{1}\right]\right)$;

(9) $\operatorname{submetacompact}\left(V=L,\left[\mathrm{~W}_{1}\right]\right)$;

(10) submetalindelöf $\left(V=L,\left[\mathrm{~B}_{1}\right]\right)$;

(11) perfect (adjoining $\omega_{2}$ random reals to a model of $V=L,\left[\mathrm{~T}_{2}\right]$ );

(12) of weight $\leq 2^{(1)}$ (by forcing, from the consistency of the existence of a weakly compact cardinal, $\left.\left[\mathrm{T}_{7}\right]\right)$;

(13) of character $<2^{\omega}$ (from PMEA [ $\mathrm{N}_{1}$ ] or by adding supercompact many Cohen reals [DTW]);

(14) of countable tightness (from PMEA, [Fr] improving a result of $\mathrm{P}$. Daniels);

(15) of character $\leq \beth_{\omega}$ (by forcing, from the consistency of the existence of infinitely many supercompact cardinals, $\left.\left[\mathrm{T}_{9}\right]\right)$.

Note that in the context of $(1)-(10)$, collectionwise normality is equivalent to paracompactness.

In various models of set theory, the following examples of normal, locally compact, not collectionwise normal spaces were known:

(a) assuming $\operatorname{MA}\left(\omega_{1}\right)$, examples may be Moore and locally countable (see $\left.\left[\mathrm{T}_{3}, \mathrm{~F}_{2}\right]\right)$ and even connected $\left[\mathrm{G}_{2}\right]$;

(b) in a model of $\mathrm{GCH}$ and $\diamond$, there is a locally countable Moore example [DS]; 
(c) assuming a combinatorial consequence $\diamond^{*}$ of $V=L$, there is a locally countable and perfect example [DG];

(d) in various models of $\mathrm{CH}$ and $\neg \mathrm{CH}$, there is an example which is locally countable, Moore, $\omega_{i}$-collectionwise Hausdorff, but not $\omega_{2}$-collectionwise Hausdorff [S];

(e) in a model of $\diamond$, there is a metalindelöf and perfect example [ $\left.\mathrm{W}_{3}\right]$.

The aim of this paper is to complete the Toronto project by proving the following result.

Theorem 0.1. If supercompact many random or Cohen reals are adjoined to a model of $Z F C$, then in the resulting model, normal locally compact spaces are collectionwise normal.

For a more detailed statement of this, see Theorem 1.1, which is stated for the generality of spaces of height $<2^{\omega}$ rather than for locally compact spaces. This means no extra complications in the proof, but te output result then also holds for some useful subclasses of spaces of height $<2^{\omega}$ such as Čech-complete spaces, $p$-spaces, and first countable spaces.

The proof of Theorem 0.1 (or 1.1 ) consists of a model-theoretic and a topological part. Forcing and reflection arguments involvi ig supercompact cardinals and even related to our theme are frequent (e.g., see $\left[\mathrm{KM}, \mathrm{F}_{6}, \mathrm{~F}_{8}\right]$ ), but our exposition of the necessary model theory follows the machinery of [DTW]. The plan of the proof is explained briefly at the beginning of $\S 1$. Note that Lemma 1.9 could have been replaced by a Nyikos-type PMEA result for random reals and a homomorphism axiom treatment à la $\left[\mathrm{F}_{6}, \mathrm{~F}_{7}\right]$ for Cohen reals.

In $\S 2$ the countably paracompact analogue of $\mathrm{NLCH}$ is treated.

The countably paracompact, locally compact space hypothesis. Every countably paracompact, locally compact space is expandable.

Note that a regular $T_{1}$ space $X$ is said to be expandable if every locally finite collection in $X$ has a locally finite expansion by open sets. If $\mathscr{F}=\left\{F_{a}: a \in A\right\}$ is an indexed collection of subsets of $X$, then $\mathscr{U}=\left\{U_{a}: a \in A\right\}$ is said to be an expansion of $\mathscr{F}$ if $F_{a} \subset U_{a}$ for every $a \in A$. A space $X$ is said to be countably paracompact if every countable open cover of $X$ has a locally finite open refinement.

Countably paracompact, locally compact spaces are known to be consistently expandable if they are

$\left(1^{\prime}\right)$ metacompact and perfect (in $\mathrm{ZFC},\left[\mathrm{A}_{2}\right]$ );

$\left(2^{\prime}\right)$ boundedly metacompact $\left(V=L\right.$ or $\left.M M,\left[\mathrm{D}_{2}, \mathrm{D}_{3}\right]\right)$;

$\left(3^{\prime}\right)$ screenable $\left(V=L\right.$ or $\left.M M,\left[\mathrm{D}_{2}, \mathrm{D}_{3}\right]\right)$;

$\left(4^{\prime}\right)$ Moore $\left(V=L,\left[\mathrm{~W}_{2}\right]\right)$;

$\left(5^{\prime}\right)$ submetacompact $\left(V=L,\left[\mathrm{~B}_{2}\right]\right)$;

$\left(6^{\prime}\right)$ of character $<2^{\omega}$ (from PMEA [Bu] or by adding supercompact many Cohen reals $\left.\left[\mathrm{T}_{4}, \mathrm{~T}_{5}\right]\right)$. 
Note that in the context of $\left(1^{\prime}\right)-\left(4^{\prime}\right)$, expandability is equivalent to paracompactness.

All the examples (a)-(e) also are examples of countably paracompact, locally compact, not expandable spaces. They are countably paracompact because they are perfectly normal. They cannot be expandable, because normal expandable spaces are collectionwise normal.

The aim of $\S 2$ is to prove

Theorem 0.2. If supercompact many random or Cohen reals are adjoined to a model of ZFC, then in the resulting model, countably paracompact and locally compact spaces are expandable.

Again, see Theorem 2.1 for a detailed statement. Also, Theorem 2.1 is formulated for the generality of spaces with height $\omega$, a class which includes Čech-complete spaces, $p$-spaces, and first countable spaces.

Our terminology and notation follow the standards of current set-theoretic topology as used in $[\mathrm{KV}]$. Our further references are $\left[\mathrm{K}_{1}\right]$ for forcing, $[\mathrm{KM}]$ for large cardinals, and $\left[\mathrm{K}_{2}\right]$ for random and Cohen reals. The ground model will be denoted by $V$. If there is no danger of confusion (and there will never be), then canonical names for elements of $V$ will be denoted by the same letter in a forcing statement. Given a set $E, \operatorname{Fn}(E, 2)$ denotes the poset of finite partial functions $E \rightarrow 2$ ordered by reverse inclusion. $M_{E}$ denotes the poset of equivalence classes of Haar measurable subsets of $2^{E}$ modulo the null sets, ordered by inclusion. If $A \subset 2^{E}$ is Haar measurable, then [A] will denote its equivalence class.

Throughout this paper, by space we shall mean a regular $T_{1}$ topological space. If $X$ is a space, $\mu \geq \omega$ is a cardinal, and $A \subset X$, then $A$ is called a $G_{\mu}$-set in $X$ if $A$ is the intersection of $\leq \mu$ open subsets of $X$. A family $\mathscr{W}$ of subsets of $X$ will be called a neighborhood base for a set $C \subset X$ if for every open set $U \supset C$ there is a $W \in \mathscr{W}$ with $C \subset \operatorname{int}(W) \subset W \subset U$. The character $\chi(C, X)$ of a subset $C$ in $X$ will be the smallest infinite cardinal $\mu$ such that $A$ has a neighborhood base of cardinality $\leq \mu$ in $X$. The height $h(X)$ will be the smallest infinite cardinal $\mu$ such that $X$ has a cover $\mathscr{C}$ by compact subsets such that $C \in \mathscr{C}$ implies $\chi(C, X) \leq \mu$ (see $\left.\left[\mathrm{A}_{1}\right]\right)$. Spaces $X$ with $h(X)=\omega$ are called spaces of point-countable type. Note that if $\chi(X)$ denotes the character of $X$, then $h(X) \leq \chi(X)$. Further, $h(X) \leq|X|$ implies $\chi(X) \leq|X|$.

\section{NORMAL, LOCALly COMPACT SPACES}

The aim of this section is to give a proof for the following theorem.

Theorem 1.1. Suppose that in $V, \kappa$ is a supercompact cardinal and $P$ is either the poset $\mathrm{Fn}(\kappa, 2)$ for adding $\kappa$ Cohen reals or the poset $M_{\kappa}$ for adding $\kappa$ random reals. Let $G$ be P-generic over $V$. Then

$V[G] \vDash$ "every normal space $X$ with $h(X)<2^{\omega}$ is collectionwise normal." 
Corollary 1.2. In the models $V[G]$ of Theorem 1.1, every normal, locally compact (or, generally, every normal Čech-complete) space is collectionwise normal.

As another special case of Theorem 1.1 the Kunen-Nyikos random real solution of the normal Moore space conjecture follows (see $\left[\mathrm{N}_{1}\right]$ and the survey $\left[\mathrm{F}_{5}\right]$ for the whole consistency proof). The Cohen real case was proved by Dow, Tall, and Weiss [DTW].

Corollary 1.3 $\left[\mathrm{N}_{1}, \mathrm{~F}_{5}\right.$, DTW]. In the models $V[G]$ of Theorem 1.1, every normal space of character $<2^{\omega}$ is collectionwise normal.

The proof of Theorem 1.1 is broken up into a sequence of lemmas. To set up the plan of the proof we first need some definitions.

We shall say that a triple $\mathfrak{X}=\langle X, r, U\rangle$ is an A-structure if

(a) $X$ is a nonvoid set;

(b) $r \subset X^{2}$ is a function from a subset of $X$ into $X$;

(c) $U \subset X^{3}$ is a ternary relation such that $\langle x, u, x\rangle \in U$ for every $x, u \in$ $X$.

Given an $A$-structure $\langle X, r, U\rangle$, let us set

(i) $Y_{a}=r^{-1}(a) \subset \operatorname{dom}(r)$ for every $a \in X$;

(ii) $Y=\operatorname{dom}(r)=\bigcup\left\{Y_{a}: a \in X\right\}$;

(iii) $U(x, u)=\{z \in X:\langle x, u, z\rangle \in U\}$ for every $\langle x, u\rangle \in X^{2}$.

The motivation is as follows.

Let $X$ be an infinite space with $h(X) \leq|X|$, and let $\mathscr{Y}=\left\{Y_{a}: a \in X\right\}$ be a closed discrete collection in $X$, indexed by points of $X$ in such a way that $Y_{a}=Y_{a^{\prime}} \neq \varnothing$ implies $a=a^{\prime}$. (Note that the empty set may be a member of $\mathscr{Y}$ and may be assigned to many indices $a$.) Let $Y=\bigcup \mathscr{Y}$. For each $x \in X$ let us fix an open neighborhood base $\mathscr{U}(x)=\{U(x, u): u \in X\}$ for $x$ in $X$ in such a way that

(1) there is a compact set $C(x)$ in $X$ such that $x \in C(x), \chi(C(x), X) \leq$ $h(X)$, and $\mathscr{U}(x)$ contains a neighborhood base for $C(x)$ in $X$, and

(2) repetitions in the indexing of $\mathscr{U}(x)$ are permitted.

Note that since the character of a space $X$ with $h(X) \leq|X|$ is not greater than $|X|$, such a neighborhood base $\mathscr{U}(x)$ always exists.

Given $X, \mathscr{Y}$, and $\{\mathscr{U}(x): x \in X\}$ as above, we can consider the $A$ structure $\mathfrak{X}=\langle X, r, U\rangle$ defined by

(A1) $\operatorname{dom}(r)=\bigcup \mathscr{Y}=Y$;

(A2) for every $y \in Y$ and $a \in X, r(y)=a$ iff $y \in Y_{a}$;

(A3) for every $x, u, z \in X,\langle x, u, z\rangle \in U$ iff $z \in U(x, u)$.

We shall say that $\mathfrak{X}=\langle X, r, U\rangle$ defined above is a relevant $A$-structure for the pair $X, \mathscr{Y}$ of a space $X$ with $h(X) \leq|X|$ and of a closed discrete collection $\mathscr{Y}=\left\{Y_{a}: a \in X\right\}$ in $X$. Note that $\mathfrak{X}$ also depends on the neighborhood system $\mathscr{U}=\{\mathscr{U}(x): x \in X\}$, but for the argument of this section it does not matter which $\mathscr{U}$ we choose provided it satisfies (1) and (2). 
Also note that (i)-(iii) are in accordance with (A1)-(A3) if $\mathfrak{X}$ is a relevant $A$-structure.

With this interpretation in mind, let us say that an $A$-structure $\mathfrak{X}=\langle X, r, U\rangle$ is separated if there is a function $f: X \rightarrow X$ such that, for every $y, y^{\prime} \in Y=$ $\operatorname{dom}(r)$,

$$
r(y) \neq r\left(y^{\prime}\right) \quad \text { implies } U(y, f(y)) \cap U\left(y^{\prime}, f\left(y^{\prime}\right)\right)=\varnothing .
$$

We shall say that $\mathfrak{X}$ is normalized if for every function $\chi: X \rightarrow 2$, there is a function $f: X \rightarrow X$ such that for every $y, y^{\prime} \in Y$,

$$
\chi(r(y)) \neq \chi\left(r\left(y^{\prime}\right)\right) \quad \text { implies } U(y, f(y)) \cap U\left(y^{\prime}, f\left(y^{\prime}\right)\right)=\varnothing .
$$

An $A$-structure $\mathfrak{X}=\langle X, r, U\rangle$ is said to be filtered if for every $x \in X$, $\{U(x, u): u \in X\}$ forms a filter-base. Relevant $A$-structures are filtered.

The proof of Theorem 1.1 will consist of a model-theoretic and a topological part. First, we shall verify that some analogues of "normal implies collectionwise normal" type results in topology (after adding random or Cohen reals) also hold for $A$-structures. The problem is that, in contrast to topology, substructures of normalized $A$-structures need not be normalized. However, the model-theoretic machinery works only if at least "many" substructures are guaranteed to be normalized. It is not quite obvious what notion of "many" is needed, on the one hand, to carry out the reflection argument (Lemma 1.13) and, on the other hand, to make sure that a relevant $A$-structure related to a normal space of height $<\mu$ possesses that many normalized substructures. The "correct" notion is that of a $(\kappa, \mu)$-c.u.b. to be defined later.

As follows from the above, the second (topological) part of the proof concentrates on producing a $(\kappa, \mu)$-c.u.b. of normalized substructures of a relevant $A$-structure. This is taken care of by introducing the notion of a splendid subset of a relevant $A$-structure related to a normal space $X$ with $h(X) \leq|X|$. Substructures on splendid subsets are filtered and normalized. Character reduction and closing up techniques guarantee that splendid subsets form a $(\kappa, \mu)$-c.u.b. (Lemma 1.20).

Having set up the plan of the proof, we can turn to the sequence of lemmas mentioned above. One of the common properties of $\operatorname{Fn}(\kappa, 2)$ and $M_{\kappa}$ that we are going to make essential use of is that they are endowed [DTW].

Definition 1.4. Assume that $E$ is an infinite set, $P$ is one of the posets $\operatorname{Fn}(E, 2)$ and $M_{E}, p \in P$, and $n \geq 2$ is an integer. For every $q \in P$ with $q \leq p$, let us define the $p$-size of $q$ by

$$
p s(q)= \begin{cases}|q \backslash p| & \text { if } P=\operatorname{Fn}(E, 2) ; \\ \max \left\{k \in \omega: \mu(q)<\frac{1}{k+1} \mu(p)\right\} & \text { if } P=M_{E} .\end{cases}
$$

Then we shall say that a family $\mathscr{L}_{n}$ of finite subsets of $P$ is an $n$-dowment below $p$, if

(i) for every maximal antichain $A$ below $p$, there is an $L \in \mathscr{L}_{n}$ with $L \subset A$; 
(ii) whenever $L_{1}, \ldots, L_{n}$ are members of $\mathscr{L}_{n}$ and $p^{\prime} \leq p$ has $p$-size $<n$, there exist $q_{i} \in L_{i}(i=1, \ldots, n)$ such that $\left\{p^{\prime}, q_{1}, \ldots, q_{n}\right\}$ has a common lower bound.

Lemma 1.5. If $E, P, p, n$ are as in Definition 1.4, then there is an $n$-dowment below $p$.

Proof. If $P=\operatorname{Fn}(E, 2)$, then this is "Dow's lemma" (see [Do ${ }_{1}$, DTW]). If $P=M_{E}$, then

$\mathscr{L}_{n}=\{L: L$ is a finite subset of conditions below $p$

$$
\text { with } \mu(\bigcup L)>(1-1 / n(n+1)) \mu(p)\}
$$

clearly satisfies both (i) and (ii).

We shall also need the following fact concerning $M_{E}$.

Proposition 1.6. Let $E$ be an infinite set, and let $p \in M_{E}$. Then there is a finite subset $D$ of $E$ such that for every pair $e, e^{\prime}$ of distinct elements of $E-D$,

$$
p^{\prime}=p \cap\left[\left\{h \in 2^{E}: h(e)=0 \text { and } h\left(e^{\prime}\right)=1\right\}\right]
$$

has $p$-size $<5$.

Proof. Take an approximation (in measure) of $p$ by a union of finitely many basic open subsets of $2^{E}$. Then the union of the supports of these finitely many open sets will do for $D$.

The next lemma is an $A$-structure analogue of the "eternity lemma" of [DTW].

Lemma 1.7. Let us suppose that in $V, \mathfrak{X}=\langle X, r, U\rangle$ is a filtered A-structure, $\rho$ is an infinite cardinal with $\rho \geq|X|$, and $P$ is one of the posets $\operatorname{Fn}(\rho, 2)$ and $M_{\rho}$. Let $G$ be P-generic over $V$, and suppose that

$$
V[G] \vDash \text { “ } \mathfrak{X} \text { is separated." }
$$

Then

$$
V \vDash \text { “ } \mathfrak{X} \text { is separated." }
$$

Proof. Without loss of generality we may assume that $X \subset \rho$. By assumption there is a function $f \in V[G]$ such that

$$
V[G] \vDash \varphi(f),
$$

where $\varphi(f)$ stands for

$$
\begin{gathered}
\text { " } f: X \rightarrow X \text { is a function and for every } y, y^{\prime} \in \operatorname{dom}(r), \\
r(y) \neq r\left(y^{\prime}\right) \text { implies } U(y, f(y)) \cap U\left(y^{\prime}, f\left(y^{\prime}\right)\right)=\varnothing . "
\end{gathered}
$$

Then there are a name $\dot{f} \in V^{P}$ and a condition $p \in G$ such that $\operatorname{val}(\dot{f}, G)=$ $f$ and

$$
p \Vdash \varphi(\dot{f}) .
$$


(Note that the free variables, other than $\dot{f}$, in $\varphi(\dot{f})$ are all from $V$ and thus can all be represented by their canonical names in the forcing statement $p \Vdash \varphi(\dot{f})$.)

Working in $V$, by Lemma 1.5 we can take a 2-dowment $\mathscr{L} \subset[P]^{<\omega}$ below $p$. For each $x \in X$, let $A(x)$ be a maximal antichain below $p$ of conditions deciding the value of $\dot{f}$ at $x$. By the definition of a 2-dowment for each $x \in X$ we can find an $L(x) \in \mathscr{L}$ with $L(x) \subset A(x)$.

Since each $L(x)$ is finite and $\mathfrak{X}$ is filtered, there is a function $g: X \rightarrow X$ in $V$ such that

$$
\begin{gathered}
\text { if } y \in \operatorname{dom}(r), q \in L(Y), u \in X, \text { and } q \Vdash \dot{f}(y)=u, \\
\text { then } U(y, g(y)) \subset U(y, u) .
\end{gathered}
$$

We shall show that $g$ defines a separation of $\mathfrak{X}$ in $V$. To see this, pick $y, y^{\prime} \in \operatorname{dom}(r)$ with $r(y) \neq r\left(y^{\prime}\right)$. We are going to show that $U(y, g(y)) \cap$ $U\left(y^{\prime}, g\left(y^{\prime}\right)\right)=\varnothing$.

Indeed, by the definition of a 2-dowment, there are $q \in L(y), q^{\prime} \in L\left(y^{\prime}\right)$ in such a way that $\left\{p, q, q^{\prime}\right\}$ has a common lower bound $r$. By $r \leq p, r \Vdash \varphi(\dot{f})$.

Let $u, u^{\prime} \in X$ be such that $q \Vdash \dot{f}(y)=u$ and $q^{\prime} \Vdash \dot{f}\left(y^{\prime}\right)=u^{\prime}$. Then by $r \leq q, q^{\prime}$ and $r \Vdash \varphi(\dot{f})$,

$$
r \Vdash U(y, u) \cap U\left(y^{\prime}, u^{\prime}\right)=\varnothing .
$$

By absoluteness, $U(y, u) \cap U\left(y^{\prime}, u^{\prime}\right)=\varnothing$. Hence, by $(*)$, we conclude that

$$
U(y, g(y)) \cap U\left(y^{\prime}, g\left(y^{\prime}\right)\right)=\varnothing .
$$

A technical consequence of Lemma 1.7 has to do with the notion of "almost separated." An $A$-structure $\mathfrak{X}=\langle X, r, U\rangle$ is called so if we can delete finitely many $Y_{a}$ 's in such a way that the remaining $A$-structure is separated. More precisely, we shall say that $\mathfrak{X}=\langle X, r, U\rangle$ is almost separated if there is a $D \in[X]^{<\omega}$ in such a way that the $A$-structure $\mathfrak{X}(D)=\langle X, r-X \times D, U\rangle$ is separated.

Corollary 1.8. Let $\mathfrak{X}, \rho, P, G$ be as in Lemma 1.7. Suppose that

$$
\begin{aligned}
V[G] & \vDash \text { “X is normalized" and } \\
V & \vDash \text { "X is almost separated." }
\end{aligned}
$$

Then

$$
V \vDash \text { “ } \mathfrak{X} \text { is separated." }
$$

Proof. Without loss of generality we may assume that $X$ is infinite. Let $D \in$ $[X]^{<\omega}$ be such that $\mathfrak{X}(D)$ is separated in $V$. Fix an $a \in X-D$ and define $\bar{r}: \operatorname{dom}(r) \rightarrow X$ by

$$
\bar{r}(y)= \begin{cases}a & \text { if } y \in \operatorname{dom}(r-X \times D), \\ r(y) & \text { otherwise. }\end{cases}
$$

Consider the filtered $A$-structure $\bar{X}=\langle X, \bar{r}, U\rangle$. Since $\mathfrak{X}$ is normalized in $V[G], \bar{X}$ is also normalized in $V[G]$. Since range $(\bar{r})$ is finite, this implies that 
$\overline{\mathfrak{X}}$ is separated in $V[G]$. By Lemma $1.7, \overline{\mathfrak{X}}$ is separated in $V$. Since both $\overline{\mathfrak{X}}$ and $\mathfrak{X}(D)$ are separated in $V$, it follows that $\mathfrak{X}$ is separated in $V$.

Lemma 1.9. Let us suppose that in $V, \mathfrak{X}=\langle X, r, U\rangle$ is a filtered A-structure, $\rho$ is an infinite cardinal with $\rho \geq|X|$, and $P$ is one of the posets $\operatorname{Fn}(\rho, 2)$ and $M_{\rho}$. Let $G$ be P-generic over $V$, and suppose that

$$
V[G] \vDash \text { “ } \mathfrak{X} \text { is normalized." }
$$

Then

$$
V \vDash \text { “ } \mathfrak{X} \text { is separated." }
$$

Proof. Without loss of generality we may assume that $X$ is infinite and $X \subset \rho$. In $V[G]$, let us define a function $\chi_{G}: \rho \rightarrow 2$ in the following way:

(1) if $P=\operatorname{Fn}(\rho, 2)$ then set $\chi_{G}=\bigcup G$;

(2) if $P=M_{\rho}$ and $\alpha \in \rho$, then set $\chi_{G}(\alpha)=i(i=0,1)$ iff $\left[\left\{h \in 2^{\rho}: h(\alpha)=i\right\}\right]^{V} \in G$.

Both of the above are standard ways of defining a "generic partition" on $\rho$; see $\left[\mathrm{K}_{2}\right.$, p. 901$]$.

Since $\mathfrak{X}$ is normalized in $V[G]$, there is an $f \in V[G]$ with

$$
V[G] \vDash \varphi(f, G),
$$

where $\varphi(f, G)$ abbreviates the statement

$$
\begin{gathered}
\text { " } f: X \rightarrow X \text { is a function and for every } y, y^{\prime} \in \operatorname{dom}(r), \\
\chi_{G}(r(y)) \neq \chi_{G}\left(r\left(y^{\prime}\right)\right) \text { implies } U(y, f(y)) \cap U\left(y^{\prime}, f\left(y^{\prime}\right)\right)=\varnothing . "
\end{gathered}
$$

Then in $V$, there is a name $\dot{f} \in V^{P}$ for $f$ and a $p \in G$ with

$$
p \Vdash \varphi(\dot{f}, \check{G}),
$$

where $\check{G}$ is the canonical name for $G$ in $V$.

Also in $V$, let $\mathscr{L} \subset[P]^{<\omega}$ be a 5-dowment below $p$.

As in the proof of Lemma 1.7, for each $x \in X$, fix an antichain $A(x)$ below $p$ of conditions deciding the value of $\dot{f}$ at $x$, pick $L(x) \in \mathscr{L}$ with $L(x) \subset A(x)$, and consider a function $g: X \rightarrow X$ in $V$ such that

$$
\begin{gathered}
\text { if } y \in \operatorname{dom}(r), g \in L(y), u \in X \text { and } q \Vdash \dot{f}(y)=u, \\
\text { then } U(y, g(y)) \subset U(y, u) .
\end{gathered}
$$

By Corollary 1.8 it is enough to show that $g$ almost separates $\mathfrak{X}$. To see this, let $D=\operatorname{dom}(p)$ if $P=\operatorname{Fn}(\rho, 2)$ and let $D \in[X]^{<\omega}$ be any set satisfying the conclusion of Proposition 1.6, if $P=M_{\rho}$. We have to show that $y, y^{\prime} \in \operatorname{dom}(r), r(y), r\left(y^{\prime}\right) \in X-D$, and $r(y) \neq r\left(y^{\prime}\right)$ imply $U(y, g(y)) \cap$ $U\left(y^{\prime}, g\left(y^{\prime}\right)\right)=\varnothing$.

Indeed, let $r(y)=a, r\left(y^{\prime}\right)=a^{\prime}$, and consider

$$
p^{\prime}=\left\{\begin{array}{l}
p \cup\left\{\langle a, 0\rangle,\left\langle a^{\prime}, 1\right\rangle\right\} \quad \text { if } P=\operatorname{Fn}(\rho, 2), \\
p \cap\left[\left\{h \in 2^{\rho}: h(a)=0 \text { and } h\left(a^{\prime}\right)=1\right\}\right] \text { if } P=M_{\rho} .
\end{array}\right.
$$


In both cases, since $a, a^{\prime} \notin D$, the $p$-size of $p$ is $<5$. Therefore there are $q \in L(y), q^{\prime} \in L\left(y^{\prime}\right)$ such that $\left\{p^{\prime}, q, q^{\prime}\right\}$ has a common lower bound $r$. By $r \leq p^{\prime} \leq p$,

$$
r \Vdash \varphi(\dot{f}, \check{G}) \quad \text { and } \quad r \Vdash \chi_{\check{G}}(r(y)) \neq \chi_{\check{G}}\left(r\left(y^{\prime}\right)\right) .
$$

Now, let $u, u^{\prime} \in X$ be such that $q \Vdash \dot{f}(y)=u$ and $q^{\prime} \Vdash \dot{f}\left(y^{\prime}\right)=u^{\prime}$. By $r \leq q, q^{\prime}$ and $(* *)$,

$$
r \Vdash U(y, u) \cap U\left(y^{\prime}, u^{\prime}\right)=\varnothing .
$$

By $(*)$ and absoluteness it follows that

$$
U(y, g(y)) \cap U\left(y^{\prime}, g\left(y^{\prime}\right)\right)=\varnothing .
$$

Supercompact elementary embeddings and $\kappa$-c.c. forcing extensions are related by the following lemma. The proof can be found in $\S 2$ of [DTW]. (Note, however, that making use of the fact that $j \mid P: P \rightarrow j(P)$ is a complete embedding for $\kappa$-c.c. posets $P$, part of their proof can be simplified to avoid master conditions and forcing products.)

Lemma 1.10. Suppose that in $V, \kappa$ is a supercompact cardinal, $P$ is a $\kappa$-c.c., separative poset, $G$ is $P$-generic over $V$, and $\beta$ is an ordinal with $\beta>\kappa+|P|$.

Then there are $M, j, G^{*}$ and $\tilde{j}$ such that the following conditions hold:

(1) $M$ is a transitive class closed under $z_{\beta}$-sequences;

(2) $j: V \rightarrow M$ is an elementary embedding with critical point $\kappa$ :

(3) $j(\kappa)>z_{\beta}$;

(4) $(M[G])_{\beta}=(V[G])_{\beta}$, i.e., $M[G]$ and $V[G]$ have the same sets of rank $<\beta$;

(5) $G^{*}$ is $j(P)$-generic over $M$;

(6) $G=\left\{p \in P: j(p) \in G^{*}\right\}$;

(7) $\tilde{j}: V[G] \rightarrow M\left[G^{*}\right]$ is an elementary embedding;

(8) if $x \in V[G]$ and $\dot{x} \in V^{P}$ is a P-name for $x$, then $\tilde{j}(x)=\operatorname{val}\left(j(\dot{x}), G^{*}\right)$;

(9) if $x \in V[G]$ has a $P$-name of cardinality $<\beth_{\beta}$ in $V$, then $\tilde{j}^{\prime \prime} x \in$ $M\left[G^{*}\right]$.

Proof. The existence and properties (1)-(8) of $M, j, G^{*}$, and $\tilde{j}$ are explicitly stated in $\S 2$ of [DTW]. To see that (9) also holds true, let us take, in $V$, a $P$ name $\tau=\left\{\left\langle\tau_{\alpha}, p_{\alpha}\right\rangle: \alpha<\mu\right\}$ for $x$ such that $\mu<\beth_{\beta}$. Then by (1), $\tilde{j}^{\prime \prime} \tau \in M$. A straightforward calculation shows that

$$
\operatorname{val}\left(j^{\prime \prime} \tau, G^{*}\right)=\tilde{j}^{\prime \prime} x
$$

so $\tilde{j}^{\prime \prime} x \in M\left[G^{*}\right]$.

Definition 1.11. Let $\kappa, \mu$ be cardinals, $X$ be a set, and suppose that $|X| \geq \kappa>$ $\mu \geq \omega$. A subset $\Delta \subset[X]^{<\kappa}$ is said to be $\mu$-directed if, for every $\Delta^{\prime} \in[\Delta]^{<\mu}$, there is a $D \in \Delta$ with $\bigcup \Delta^{\prime} \subset D$. A subset $\Gamma \subset[X]^{<\kappa}$ is called $(\kappa, \mu)$-closed, if for every $\mu$-directed $\Delta \in[\Gamma]^{<\kappa}, \cup \Delta \in \Gamma$; cofinal, if for every $Z \in[X]^{<\kappa}$ there is a $D \in \Gamma$ with $Z \subset D$. 
If $\Gamma \subset[X]^{<\kappa}$ is both $(\kappa, \mu)$-closed and cofinal, then we shall say that $\Gamma$ is a $(\kappa, \mu)-c . u . b$.

Remark. By the definition above, $(\kappa, \omega)$-c.u.b.'s are exactly the c.u.b.'s in the usual sense. For $\mu \geq \omega_{1}$, ordinary c.u.b.'s are $(\kappa, \mu)$-c.u.b.'s, but not vice versa, as is shown by the example $\Gamma=\left\{Z \in[\lambda]^{<\kappa}: \operatorname{cf}(\sup Z) \geq \mu\right\}$, where $X=\lambda \geq \kappa>\mu \geq \omega_{1}$.

An ascending chain $\Delta \in[X]^{<\kappa}$ of cofinality $\delta$, where $\mu \leq \delta<\kappa$, is clearly $\mu$-directed. There arises the question of whether, in the above definition, one can relax the requirement that $\Gamma$ is closed under unions of $\mu$-directed sets to closedness under unions of such ascending chains only and still get the same concept. The following simple example (given by F. Galvin) shows that this is not so.

Let $\mu=\omega_{1}, \aleph_{\omega}^{\omega}<\operatorname{cf}(\kappa)=\kappa<\lambda$, and let us put $\Gamma=\left\{Z \in[\lambda]^{<\kappa}:|Z| \neq \aleph_{\omega}\right\}$. Then $\Gamma$ is cofinal in $[\lambda]^{<\kappa}$ and, for each regular $\delta$ with $\omega<\delta<\kappa, \Gamma$ is closed under ascending unions of cofinality $\delta$. On the other hand, $\Delta=\left[\aleph_{\omega}\right]^{\omega} \in[\Gamma]^{<\kappa}$ is $\omega_{1}$-directed, but $\bigcup \Delta=\aleph_{\omega} \notin \Gamma$.

An important observation concerning $\mu$-directedness is that it is preserved by c.c.c. forcing.

Proposition 1.12. Suppose that in $V, \kappa>\mu \geq \omega$ are cardinals, $P$ is a c.c.c. poset, $X$ is a set with $|X| \geq \kappa$, and $\Delta \subset[X]^{<\kappa}$ is $\mu$-directed. Let $G$ be $P$-generic over $V$. Then

$$
V[G] \vDash " \Delta \text { is } \mu \text {-directed." }
$$

Proof. In $V[G]$, let $\Delta^{\prime} \in[\Delta]^{<\mu}$. Since $P$ is c.c.c, there is a $\Delta^{\prime \prime} \in[\Delta]^{<\mu}$ in $V$ such that $\Delta^{\prime} \subset \Delta^{\prime \prime}$. Since $\Delta$ was $\mu$-directed in $V$, there is a $D \in \Delta$ satisfying $\cup \Delta^{\prime \prime} \subset D$, and thus $\cup \Delta^{\prime} \subset D$.

In the following lemma, let $\Phi(\mathfrak{X})$ stand for " $\mathfrak{X}$ is not separated."

Lemma 1.13. Suppose that in $V, \kappa$ is a supercompact cardinal, $\mu$ is a cardinal with $\kappa>\mu \geq \omega, P$ is either the poset $\operatorname{Fn}(\kappa, 2)$ or the poset $M_{\kappa}$, and $G$ is $P$-generic over $V$. Then

$V[G] \vDash$ "If $\chi=\langle X, r, U\rangle$ is an A-structure, $|X| \geq \kappa, \Gamma$ is a $(\kappa, \mu)$-c.u.b. subset of $[X]^{<\kappa}$, and $\Phi(\mathfrak{X})$ holds, then there is a $Z \in \Gamma$ such that $\Phi\left(\left\langle Z, r \cap Z^{2}, U \cap Z^{3}\right\rangle\right)$ holds."

Proof. Without loss of generality we may assume that the underlying set $X$ of $\mathfrak{X}$ is an ordinal $\lambda \geq \kappa$. Let us choose another ordinal $\beta$ such that $\beta>\lambda+\omega$. With this $\beta$, let us consider $M, j, G^{*}$, and $\tilde{j}$ such as in the conclusion of Lemma 1.10. Throughout the proof, the notation $(k), k=1, \ldots, 9$, will refer to one of the conditions (1)-(9) in Lemma 1.10 .

Since $\beta>\lambda+\omega, \Phi(\mathfrak{X})$ and " $\Gamma$ is $\mu$-directed" are ZFC-equivalent to their versions with quantifiers restricted to sets of rank $<\beta$.

Note that in $V[G], \Gamma$ is $\mu$-directed (since it is cofinal in $[\lambda]^{<\kappa}$ ). Therefore

$$
(V[G])_{\beta} \vDash \Phi(\mathfrak{X}) \text { and " } \Gamma \text { is } \mu \text {-directed." }
$$


By (4),

$$
(M[G])_{\beta} \vDash \Phi(\mathfrak{X}) \quad \text { and " } \Gamma \text { is } \mu \text {-directed." }
$$

Again, since $\Phi(\mathfrak{X})$ and " $\Gamma$ is $\mu$-directed" are ZFC-equivalent to their versions restricted to sets of rank $<\beta$, it follows that

$$
M[G] \vDash \Phi(\chi) \text { and " } \Gamma \text { is } \mu \text {-directed." }
$$

By standard factorization properties of $P=\operatorname{Fn}(j(\kappa), 2)$ or $M_{j(\kappa)}$ (see $\left[\mathrm{K}_{2}\right.$, p. 904]), $M\left[G^{*}\right]$ is an extension of $M[G]$ by forcing with $\operatorname{Fn}(j(\kappa)-\kappa, 2)$ and $M_{j(\kappa)-\kappa^{\prime}}$, respectively. Thus

(a) $M\left[G^{*}\right] \vDash \Phi(\mathfrak{X})$ by Lemma 1.7 ;

(b) $M\left[G^{*}\right] \vDash$ " $\Gamma$ is $\mu$-directed" by Proposition 1.12 .

Let $\tilde{\mathfrak{X}}=\left\langle\tilde{j}^{\prime \prime} \lambda, \tilde{j}^{\prime \prime} r, \tilde{j}^{\prime \prime} U\right\rangle$ and $\tilde{\Gamma}=\tilde{j}^{\prime \prime} \Gamma$. Note that in $V, \lambda, r, U$, and $\Gamma$ can all be represented by names of cardinality $<\beth \beta$. (For $\lambda, r$, and $U$ this is trivial; for $\Gamma$ see $\S 5$ of $\left[\mathrm{F}_{8}\right]$.) Thus by (9), $\widetilde{\mathfrak{X}}, \widetilde{\Gamma} \in M\left[G^{*}\right]$.

Further, since $\tilde{j} \uparrow X$ is an isomorphism between $\mathfrak{X}$ and $\tilde{\mathfrak{X}}$, it follows from (a) that

(a') $M\left[G^{*}\right] \vDash \Phi(\widetilde{\mathfrak{X}})$.

Since in $V[G], A \in \Gamma, A \in \Gamma$ implies $|A|<\kappa$, and $j(\alpha)=\alpha$ for every $\alpha \in \kappa$, if follows from (7) that $\tilde{j}(A)=\tilde{j}^{\prime \prime} A$. Thus $\widetilde{\Gamma}=\tilde{j}^{\prime \prime} \Gamma=\left\{\tilde{j}^{\prime \prime} A: A \in \Gamma\right\}$, so by (b),

$\left(\mathrm{b}^{\prime}\right) \quad M\left[G^{*}\right] \vDash$ " $\widetilde{\Gamma}$ is $\mu$-directed."

By elementarity of $\tilde{j}$ and $\tilde{j}(\mu)=\mu$,

(c) $M\left[G^{*}\right] \vDash \Phi(\tilde{j}(\mathfrak{X}))$ and “ $\tilde{j}(\Gamma)$ is a $(\tilde{j}(\kappa), \mu)$-c.u.b. on $[\tilde{j}(\lambda)]^{<\tilde{j}(\kappa)}$. ."

By $\widetilde{\Gamma}=\tilde{j}^{\prime \prime} \Gamma \subset \tilde{j}(\Gamma)$ and $\tilde{j}(\kappa)=j(\kappa)>z_{\beta}^{V}>|\widetilde{\Gamma}|$,

(d) $M\left[G^{*}\right] \vDash$ " $\widetilde{\Gamma}$ is a $\mu$-directed subset of $\tilde{j}(\Gamma)$ of cardinality $<\tilde{j}(\kappa)$."

Since $U \Gamma=\lambda$, it follows from (c) and (d) that

$$
M\left[G^{*}\right] \vDash \text { “ } \tilde{j}^{\prime \prime} \lambda=\bigcup \tilde{\Gamma} \in \tilde{j}(\Gamma) . "
$$

Therefore

(e) $M\left[G^{*}\right] \vDash$ “ $\tilde{j}(\mathfrak{X})$ has a substructure $\widetilde{\mathfrak{X}}$ with underlying set in $\tilde{j}(\Gamma)$ such that $\Phi(\tilde{\mathfrak{X}})$ holds."

By the elementarity of $\tilde{j}$, (e) can be pulled back to get

$$
V[G] \vDash \text { "there is a } Z \in \Gamma \text { such that } \Phi\left(\left\langle Z, r \cap Z^{2}, U \cap Z^{3}\right\rangle\right) \text { holds." }
$$

Definition 1.14. Let $\kappa, \nu$ be cardinals with $\omega \leq \nu<\nu^{+} \leq \operatorname{cf}(\kappa), X$ be a normal space with $|X| \geq \kappa$ and $h(X) \leq \nu, \mathscr{Y}=\left\{Y_{a}: a \in X\right\}$ be a closed discrete collection in $X, Y=\bigcup \mathscr{Y}$, and let us fix a relevant $A$-structure $\mathfrak{X}=\langle X, r, U\rangle$ for $X$ and $\mathscr{Y}$. For every subset $Z$ of $X$ and every point $x \in Z$, let

$$
C(x, Z)=\bigcap\{\overline{U(x, u)}: u \in Z\},
$$


and for every $a \in X$, let

$$
Y_{a}(Z)=\bigcup\left\{C(y, Z): y \in Y_{a} \cap Z\right\} .
$$

(Note that since $Y_{a} \cap Z$ may be empty, some of the $Y_{a}(Z)$ 's may also be empty.)

By normality, for every $a \in X$ fix a pair of open sets $G(a)$ and $G^{c}(a)$ in such a way that

$$
Y_{a} \subset G(a), \quad Y-Y_{a} \subset G^{c}(a), \quad \text { and } \quad \overline{G(a)} \cap \overline{G^{c}(a)}=\varnothing
$$

hold.

Then we shall say that a subset $Z \subset X$ is splendid if the following conditions are satisfied:

(a) for every $a \in X$,

$$
Y_{a}(Z) \subset G(a) \cap \bigcap\left\{G^{c}(b): Y_{b} \cap Z \neq \varnothing \text { and } b \neq a\right\} ;
$$

(b) $\mathscr{Y}(Z)=\left\{Y_{a}(Z): a \in X\right\}$ is a discrete collection of subsets in $X$;

(c) for every $x \in Z, C(x, Z)$ is a compact subset of $X$ and $\{U(x, u)$ : $u \in Z\}$ is a neighborhood base for $C(x, Z)$ in $X$.

Definition $1.14^{\prime}$. Let $Z \in[X]^{<\kappa}$. We shall say that a set $Z^{\prime} \in[X]^{<\kappa}$ is an extension of $Z$ (toward splendidness) if $Z^{\prime} \supset Z$ and the following conditions hold:

$\left(\mathrm{a}^{\prime}\right)$ for every $a \in X$,

$$
\begin{aligned}
& Y_{a}\left(Z, Z^{\prime}\right)=\bigcup\left\{C\left(y, Z^{\prime}\right): y \in Y_{a} \cap Z\right\} \\
& \subset G(a) \cap \bigcap\left\{G^{C}(b): Y_{b} \cap Z \neq \varnothing \text { and } b \neq a\right\} ;
\end{aligned}
$$

$\left(\mathbf{b}^{\prime}\right) \mathscr{Y}\left(Z, Z^{\prime}\right)=\left\{Y_{a}\left(Z, Z^{\prime}\right): a \in X\right\}$ is a discrete collection of sets in $X$;

$\left(c^{\prime}\right)$ for every $x \in Z$, there is a compact set $C\left(x, Z, Z^{\prime}\right)$ such that $C\left(x, Z^{\prime}\right)$ $\subset C\left(x, Z, Z^{\prime}\right) \subset C(x, Z)$ and $\left\{U(x, u): u \in Z^{\prime}\right\}$ contains a neighborhood base for $C\left(x, Z, Z^{\prime}\right)$ in $X$.

Proposition 1.15. With the definitions above, if $Z$ is a splendid subset of $X$, then the substructure $\left\langle Z, r \cap Z^{2}, U \cap Z^{3}\right\rangle$ of $\mathfrak{X}$ is filtered and normalized.

Proof. This follows immediately from (b), (c) in the definition of "splendid" and the normality of $X$.

Proposition 1.16. In a normal space $X$, every discrete collection $\mathscr{Y}=$ $\left\{Y_{a}: a \in X\right\}$ has a discrete expansion $\mathscr{R}=\left\{R_{a}: a \in X\right\}$ by $G_{\mu}$-sets, where $\mu=|\mathscr{Y}|+\omega=\left|\left\{a \in X: Y_{a} \neq \varnothing\right\}\right|+\omega$.

Proof. By normality, for each $a \in X$ there is an open set $V_{a}$ in $X$ such that $V_{a} \supset Y_{a}, \bar{V}_{a} \cap \bigcup\left\{Y_{b}: b \in X-\{a\}\right\}=\varnothing$, and $Y_{a}=\varnothing$ implies $V_{a}=\varnothing$. Also by normality, there is an open set $W$ with

$$
\bigcup \mathscr{Y} \subset W \subset \bar{W} \subset \bigcup\left\{V_{a}: a \in X\right\} .
$$


Then $\mathscr{R}=\left\{R_{a}: a \in X\right\}$, where $R_{a}=W-\bigcup\left\{\bar{V}_{b}: b \in X-\{a\}\right\} \quad(a \in X)$, will be as required.

To prove Lemma 1.18, we shall need the following standard facts from topology. The straightforward proofs are left to the reader.

Proposition 1.17. The following assertions are valid for a space $X$ :

(1) if $C \subset X$ is compact, $\mathscr{W}$ is a neighborhood base for $C$ in $X$, and $\mathscr{K}$ is a locally finite family in $X$, then there is a $W \in \mathscr{W}$ such that $W$ meets only finitely many members of $\mathscr{K}$;

(2) if $\mathscr{C}$ is a filter-base of compact subsets of $X$ and for each $C \in \mathscr{C}, \mathscr{W}(C)$ is a neighborhood base for $C$, then $\bigcup\{\mathscr{W}(C): C \in \mathscr{C}\}$ is a neighborhood base for $\cap \mathscr{C}$;

(3) If $x \in X, C \ni x$ is a compact subset of a regular $X$ with $\chi(C, X) \leq \mu$, $\mathscr{W} \subset \mathscr{P}(X)$ contains a neighborhood base for both $x$ and $C$ in $X$, and $R$ is a $G_{\mu}$-subset containing $x$, then there is a $\mathscr{W}^{\prime} \subset \mathscr{W}$ such that $\left|\mathscr{W}^{\prime}\right| \leq \mu$, $C^{\prime}=\bigcap\left\{\bar{W}: W \in \mathscr{W}^{\prime}\right\} \subset C \cap R$, and $\mathscr{W}^{\prime}$ forms a neighborhood base for $C^{\prime}$ in $X$.

Lemma 1.18. Under the assumptions of Definitions 1.14 and $1.14^{\prime}$, every $Z \in$ $[X]^{<\kappa}$ has an extension $Z^{\prime} \in[X]^{<\kappa}$.

Proof. Let $Z \in[X]^{<\kappa}$. Without loss of generality we may assume that $|Z|=$ $\rho>\omega$. Let us put $D(Z)=\left\{a \in X: Y_{a} \cap Z=\varnothing\right\}$. Observe that $|D(Z)| \leq \rho$.

By Proposition 1.16, $Y(Z)=\left\{Y_{a} \cap Z: a \in X\right\}$ has a discrete expansion $R=$ $\left\{R_{a}: a \in X\right\}$ by $G_{\rho}$-sets in $X$. Since $|D(Z)| \leq \rho$, without loss of generality we may assume that

$$
a \in D(Z) \text { implis } R_{a} \subset G(a) \cap \bigcap\left\{G^{c}(b): b \in D(Z) \text { and } b \neq a\right\} .
$$

By Proposition 1.17(3), for each $x \in Z$ we can pick a compact set $C(x)$ with $C(x, Z) \supset C(x) \ni x$ such that $C(x)$ has a neighborhood base $\{U(x, u): u \in$ $\left.Z_{x}\right\}$ of cardinality $\leq \rho \cdot \nu$, and if $a \in D(Z)$ and $x \in Y_{a} \cap Z$, then $C(x) \subset$ $R_{a}$ holds. Let us put $Z^{\prime}=Z \cup \cup\left\{Z_{x}: x \in Z\right\}$ and let, for every $x \in Z$, $C\left(x, Z, Z^{\prime}\right)$ be the compact set $C(x)$ picked above. This choice of $Z^{\prime}$ and $C\left(x, Z, Z^{\prime}\right)$ clearly satisfies $\left(\mathrm{a}^{\prime}\right),\left(\mathrm{b}^{\prime}\right)$, and $\left(\mathrm{c}^{\prime}\right)$ in Definition $1.14^{\prime}$.

Lemma 1.19. Under the assumptions of Definitions 1.14 and $1.14^{\prime}$, let $\Delta \subset$ $[X]^{<\kappa}$ be a $\nu^{+}$-directed family such that every $Z \in \Delta$ has an extension $Z^{\prime} \in \Delta$. Then $Z^{*}=\bigcup \Delta$ is a splendid subset of $X$.

Proof. To see that (a) is true, let $a \in X$ and let $y$ be an arbitrary point of $Y_{a} \cap Z$. Then there is a $Z \in \Delta$ with $y \in Y_{a} \cap Z$. Let $Z^{\prime} \in \Delta$ be an extension of $Z$. Then by $\left(\mathrm{a}^{\prime}\right)$,

(i) $C\left(y, Z^{*}\right) \subset C\left(y, Z^{\prime}\right) \subset Y_{a}\left(Z, Z^{\prime}\right) \subset G(a)$.

Further, let $b \neq a$ be such that $Y_{b} \cap Z^{*} \neq \varnothing$. Then there is a $Z_{1} \in \Delta$ with $y \in Z_{1}$ and $Y_{b} \cap Z_{1} \neq \varnothing$. Let $Z_{1}^{\prime} \in \Delta$ be an extension of $Z_{1}$. Then, again by $\left(a^{\prime}\right)$ of Definition $1.14^{\prime}$, it follows that 
(ii) $C\left(y, Z^{*}\right) \subset C\left(y, Z_{1}^{\prime}\right) \subset Y_{a}\left(Z_{1}, Z_{1}^{\prime}\right) \subset G^{c}(b)$.

Since $b \neq a$ was arbitrary, it follows from (i) and (ii) that

$$
C\left(y, Z^{*}\right) \subset G(a) \cap \bigcap\left\{G^{c}(b): Y_{b} \cap Z^{*} \neq \varnothing \text { and } b \neq a\right\} .
$$

As $y \in Y_{a} \cap Z^{*}$ was arbitrary, this implies

$$
Y_{a}\left(Z^{*}\right) \subset G(a) \cap \bigcap\left\{G^{c}(b): Y_{b} \cap Z^{*} \neq \varnothing \text { and } b \neq a\right\} .
$$

To see that $Z^{*}$ also satisfies (b), note first that since $Z^{*}$ has already been shown to satisfy (a), $\left\{\overline{Y_{a}\left(Z^{*}\right)}: a \in X\right\}$ is a pairwise disjoint collection of closed sets in $X$. So in order to prove that $\mathscr{Y}\left(Z^{*}\right)$ is a discrete collection it is enough to show that $\mathscr{Y}\left(Z^{*}\right)$ is locally finite. To see local finiteness, let $x$ be an arbitrary point in $X$. Since $h(X) \leq \nu$, there is a compact subset $C \ni x$ of $X$ such that $C$ has a neighborhood base $\mathscr{W}$ of cardinality $\leq \nu$ in $X$. Making use of Proposition 1.1.7(1), suppose indirectly that for every $W \in \mathscr{W}$, there is a countably infinite subset $A(W) \subset X$ such that $a \in A(W)$ implies $W \cap Y_{a}\left(Z^{*}\right) \neq$ $\varnothing$.

Then for each $W \in \mathscr{W}$ and $a \in A(W)$, let us pick a point $y(a, W) \in Y_{a} \cap Z^{*}$ such that $C\left(y(a, W), Z^{*}\right) \cap W \neq \varnothing$. Since $\Delta$ is $\nu^{+}$-directed, there a $Z \in \Delta$ with

$$
\{y(a, W): W \in \mathscr{W} \text { and } a \in A(W)\} \subset Z .
$$

Let $Z^{\prime} \in \Delta$ be an extension of $Z$. Then by $C\left(y(a, W), Z^{*}\right) \subset C\left(y(a, W), Z^{\prime}\right)$ it follows that for every $W \in \mathscr{W}, W$ meets $Y_{a}\left(Z, Z^{\prime}\right)$ for infinitely many $a \in X$, in contradiction with $\left(\mathrm{b}^{\prime}\right)$ and (1) of Proposition 1.17.

Finally, to see that $Z^{*}$ satisfies (c), fix an extension $Z^{\prime} \in \Delta$ for each $Z \in \Delta$ and let us observe that for every $x \in Z^{*}$

$$
C\left(x, Z^{*}\right)=\bigcap\left\{C\left(x, Z, Z^{\prime}\right): Z \in \Delta\right\} .
$$

Since each $C\left(x, Z, Z^{\prime}\right), Z \in \Delta$, is compact, $C\left(x, Z^{*}\right)$ is compact in $X$. Since $\Delta$ was directed, $\left\{C\left(x, Z, Z^{\prime}\right): Z \in \Delta\right\}$ forms a filter-base. Then by Proposition 1.17(2),

$$
\left\{U(y, u): u \in Z^{*}\right)=\bigcup_{Z \in \Delta}\{U(y, u): u \in Z\}
$$

is a neighborhood base for $C\left(y, Z^{*}\right)$.

Remark. Note that we have used that $h(x, X)<\nu^{+}$for all $x \in X$, in contrast to $\left[\mathrm{N}_{1}\right.$, DTW], which use $\chi(y, X)<2^{\omega}$ only for $y \in \bigcup \mathscr{Y}$.

Lemma 1.20. Under the assumptions of Definition 1.14, the set

$$
\Gamma=\left\{Z \in[X]^{<\kappa}: Z \text { is splendid }\right\}
$$

is $a\left(\kappa, \nu^{+}\right)$-c.u.b. on $[X]^{<\kappa}$.

Proof. In order to prove that $\Gamma$ is $\left(\kappa, \nu^{+}\right)$-closed, let us consider a $\nu^{+}$-directed subset $\Delta \in[\Gamma]^{<\kappa}$, and let us put $Z^{*}=\bigcup \Delta$. Since $\kappa$ is regular, $Z^{*} \in[X]^{<\kappa}$. 
Note that if $Z \in[X]^{<\kappa}$ is splendid, then $Z$ extends itself. Thus by Lemma $1.19, Z^{*}$ is splendid and $Z^{*} \in \Gamma$.

In order to see that $\Gamma$ is cofinal in $[X]^{<\kappa}$, let $Z$ be an arbitrary member of $\Gamma$, and let us define, inductively, a sequence $\left\{Z_{\xi}: \xi \in \nu^{+}\right\}$by putting

$$
\begin{aligned}
& Z_{0}=Z ; \\
& Z_{\xi+1}=Z_{\xi}^{\prime}, \text { where } Z_{\xi}^{\prime} \text { is as in Lemma } 1.18 \\
& Z_{\xi}=\bigcup\left\{Z_{\eta}: \eta<\xi\right\}, \text { if } \xi<\nu^{+} \text {is a limit ordinal. }
\end{aligned}
$$

Since $\kappa=\operatorname{cf}(\kappa)>\nu^{+}, Z^{*}=\bigcup\left\{Z_{\xi}: \xi \in \nu^{+}\right\} \in[X]^{<\kappa}$. By Lemma 1.19, $Z^{*}$ is splendid.

Proof of Theorem 1.1. Suppose indirectly that in $V[G]$ there is a normal space $X$ such that $h(X)=\nu<2^{\omega}=\kappa$ and there is an unseparated discrete collection $\mathscr{Y}=\left\{Y_{a}: a \in X\right\}$ of closed subsets. By adding a clopen discrete subspace of size $\kappa$ to $X$ if necessary, without loss of generality we may assume that $|X| \geq \kappa$. Consider a relevant $A$-structure $\mathfrak{X}=\langle X, r, U\rangle$ for the pair $X, \mathscr{Y}$. By Lemma 1.20 and Proposition 1.15,

$$
\left\{Z \in[X]^{<\kappa}:\left\langle Z, r \cap Z^{2}, U \cap Z^{3}\right\rangle \text { is filtered and normalized }\right\}
$$

contains a $\left(\kappa, \nu^{+}\right)$-c.u.b. $\Gamma$. Since $\mathscr{Y}$ was an unseparated collection in the topological space $X, \mathfrak{X}$ is an unseparated $A$-structure in $V[G]$. Thus by Lemma 1.13, there is $Z \in \Gamma$ such that $\left\langle Z, r \cap Z^{2}, U \cap Z^{3}\right\rangle$ is an unseparated $A$ structure in $V[G]$. On the other hand, it follows from Lemma 1.9 and standard factorization properties of $P$ (see $\left[\mathrm{K}_{2}\right.$, p. 904]) that in $V[G]$ every filtered and normalized $A$-structure of cardinality $<\kappa$ is separated, in contradiction with the existence of $Z$ as above.

\section{The COUNTABLy PARACOMPACT CASE}

This section is devoted to the proof of the following result.

Theorem 2.1. Suppose that in $V, \kappa$ is a supercompact cardinal and $P$ is either the poset $\mathrm{Fn}(\omega \times \kappa, 2)$ for adding $\kappa$ Cohen reals or the poset $M_{\omega \times \kappa}$ for adding $\kappa$ random reals. Let $G$ be P-generic over $V$. Then

$V[G] \vDash$ "every countably paracompact space with $h(X)=\omega$ is expandable."

Corollary 2.2. In the models $V[G]$ of Theorem 2.1 , every countably paracompact, locally compact (or, more generally, every countably paracompact, Čechcomplete) space is expandable.

Another special case of Theorem 2.1 is Burke's (and Tall's) solution to the "countably paracompact Moore space conjecture." More exactly, the following result was proved first by Burke $[\mathrm{Bu}]$ for random reals and then by Tall $\left[\mathrm{T}_{4}, \mathrm{~T}_{5}\right]$ for Cohen reals. (Note that in $\left[\mathrm{Bu}, \mathrm{T}_{4}, \mathrm{~T}_{5}\right]$ the authors restrict themselves to discrete rather than, more generally, locally finite collections, but the two are equivalent by the Smith-Krajewski theorem quoted below.) 
Corollary 2.3 $\left[\mathrm{Bu}, \mathrm{T}_{4}, \mathrm{~T}_{5}\right]$. In the models $V[G]$ of Theorem 2.1, every countably paracompact, first countable space is expandable.

In countably paracompact spaces expandability is equivalent to the apparently weaker notion of discrete $\theta$-expandability. A space $X$ is called discretely $\theta$-expandable $[\mathrm{Sm}]$ if every closed discrete family $\left\{Y_{a}: a \in X\right\}$ has a sequence of expansions $\mathscr{G}_{n}=\{G(n, a): a \in X\} \quad(n \in \omega)$ is such a way that for every $x \in X$, there is an $n=n(x) \in \omega$ such that $\mathscr{G}_{n}$ is locally finite at $x$. We can invoke the following result from topology [SmK, Sm]:

The Smith-Krajewski theorem. A countably paracompact space is expandable if and only if it is discretely $\theta$-expandable.

The proof of Theorem 2.1 will follow the plan of the proof of Theorem 1.1. Several results used will actually be the same. We shall spell out those that are different following the numbering in the first section.

The definition of an $A$-structure and of a relevant $A$-structure for a pair $X$, $\mathscr{Y}$ will remain the same. Let us say that an $A$-structure $\mathfrak{X}=\langle X, r, U\rangle$ is $\theta$-expandable if there is a sequence $f_{n}: X \rightarrow X \quad(n \in \omega)$ of functions such that for every $x \in X$ there are $t \in X, n \in \omega$ with

$$
S\left(x, t, f_{n}\right)=\left\{r(y): y \in \operatorname{dom}(r) \text { and } U(x, t) \cap U\left(y, f_{n}(y)\right) \neq \varnothing\right\}
$$

finite.

We shall say that $\mathfrak{X}$ is $\omega$-expandable if, for every function $\chi: X \rightarrow \omega$, there is a function $f: X \rightarrow X$ such that for every $x \in X$ there is a $t \in X$ with

$$
T(x, t, \chi, f)=\{\chi(r(y)): y \in \operatorname{dom}(r) \text { and } U(x, t) \cap U(y, f(y)) \neq \varnothing\}
$$
finite.

To motivate this notion, let $X$ be countably paracompact, $\mathfrak{X}$ be relevant for $X, \mathscr{Y}$. Then " $\mathfrak{X}$ is $\omega$-expandable" says that any partition $\chi: X \rightarrow \omega$ of (the indices of members of) $\mathscr{Y}$ into $\omega$ many pieces has a locally finite open expansion.

The definition of a filtered $A$-structure remains the same.

Definition 2.4 and Lemma 2.5 are identical with Definition 1.4 and Lemma 1.5. Instead of Proposition 1.6 we need the following fact with a similar proof.

Proposition 2.6. Let $E$ be an infinite set, let $p \in M_{E}$, and let $m \in \omega$. Then there is a finite subset $D$ of $E$ such that for every sequence.

$$
\begin{aligned}
& \left\{\left\langle e_{0}, i_{0}\right\rangle,\left\langle e_{1}, i_{1}\right\rangle, \ldots\left\langle e_{m}, i_{m}\right\rangle\right\} \subset(E-D) \times 2, \\
& p^{\prime}=p \cap\left[\left\{h \in 2^{E}: h\left(e_{j}\right)=i_{j}, j=0, \ldots, m\right\}\right]
\end{aligned}
$$

has $p$-size $<2^{m+1}+2$.

The analogue of Lemma 1.7 is this. 
Lemma 2.7. Let us suppose that in $V, \mathfrak{X}=\langle X, r, U\rangle$ is a filtered A-structure, $\rho$ is a cardinal with $\rho \geq|X|$, and $P$ is one of the posets $\operatorname{Fn}(\omega \times \rho, 2)$ and $M_{\omega \times \rho}$. Let $G$ be P-generic over $V$, and suppose that

$$
V[G]=\text { " } \mathfrak{X} \text { is } \theta \text {-expandable". }
$$

Then

$$
V \vDash \text { “ } \mathfrak{X} \text { is } \theta \text {-expandable". }
$$

Proof. Without loss of generality we may assume that $X$ is infinite and $X \subset \rho$. By assumption there is an $f \in V[G]$ such that $V[G] \vDash \varphi(f)$, where $\varphi(f)$ stands for

" $f: \omega \times X \rightarrow X$ is a function, $y \in Y$ and $n \in \omega$ imply $U(y, f(n+1, y)) \subset$ $U(y, f(n, y))$, and further, for every $x \in X$ there are $t \in X$ and $n \geq 2$ such that

$$
S(x, t, n, f)=\{r(y): y \in \operatorname{dom}(r) \text { and } U(x, t) \cap U(y, f(n, y)) \neq \varnothing\}
$$

is finite."

Then there are an $\dot{\mathfrak{f}} \in V^{P}$ and a $p \in G$ with $\operatorname{val}(\dot{\mathfrak{f}}, G)=f$ and $p \Vdash \varphi(\dot{\mathfrak{f}})$.

Working in $V$, for each $n \geq 2$, let $\mathscr{L}_{n} \subset[P]^{<\omega}$ be an $n$-dowment below $p$. For each $z \in X$ and $n \geq 2$, let $A(n, z)$ be a maximal antichain below $p$ of conditions deciding the value of $\dot{f}$ at $\langle n, z\rangle$. For each $n \geq 2$ and $z \in X$, fix an $L(n, z) \in \mathscr{L}_{n}$ with $L(n, z) \subset A(n, z)$. Since each $L(n, z)$ is finite and $\mathfrak{X}$ is filtered, for every $n \geq 2$ we can take a function $g_{n}: X \rightarrow X$ in $V$ such that

$$
\text { if } \begin{gathered}
y \in \operatorname{dom}(r), \\
\text { then } U(y, g(y)) \subset U(y, u) .
\end{gathered}
$$

We are going to show that $\left\{g_{n}: n \geq 2\right\}$ witnesses $\theta$-expandability of $\mathfrak{X}$ in $V$. To see this, let $x \in X$. By $p \Vdash \varphi(\dot{f})$ there are $t \in X, n \geq 2, k \in \omega$, and $p(x) \leq p$ with $p(x) \Vdash|S(x, t, n, \dot{\mathfrak{f}})| \leq k$.

Since $\varphi(f)$ implies that $S(x, t, n, f)$ decreases as $n$ increases, without loss of generality we may assume that $n>p s(p(x))+k+2$.

Indirectly assume that $\left|S\left(x, t, g_{n}\right)\right| \geq \omega$. Then there is a sequence $y_{0}, \ldots$, $y_{k} \in \operatorname{dom}(r)$ such that

(i) $r\left(y_{i}\right)=a_{i}(i=0, \ldots, k)$ are pairwise distinct;

(ii) $U(x, t) \cap U\left(y_{i}, g_{n}\left(y_{i}\right)\right) \neq \varnothing$ for $i=0, \ldots, k$.

Since $\mathscr{L}_{n}$ was an $n$-dowment, there are $q_{i} \in L\left(n, y_{i}\right)(i=0, \ldots, k)$ such that $\left\{p(x), q_{0}, \ldots, q_{k}\right\}$ has a common lower bound $r$. By $r \leq p(x)$,

$$
r \Vdash|S(x, t, n, \mathfrak{f})| \leq k .
$$

On the other hand, let $u_{i} \in X \quad(i=0, \ldots, k)$ be such that $q_{i} \Vdash \dot{f}\left(n, y_{i}\right)=u_{i}$ $(i=0, \ldots, k)$. By $(*)$ and (ii) it follows that

$$
U(x, t) \cap U\left(y_{i}, u_{i}\right) \neq \varnothing \quad(i=0, \ldots, k) .
$$

By absoluteness, this implies $r \Vdash \mid S(x, t, n, \dot{\mathfrak{f}} \mid \geq k+1$, in contradiction with $(* *)$. 
The equivalent of Corollary 1.8 is not needed for the countably paracompact case. The analogue of Lemma 1.9 is this.

Lemma 2.9. Let us suppose that in $V, \mathfrak{X}=\langle X, r, U\rangle$ is a filtered A-structure, $\rho$ is a cardinal with $\rho \geq|X|$, and $P$ is one of the posets $\operatorname{Fn}(\omega \times \rho, 2)$ and $M_{\omega \times \rho}$. Let $G$ be P-generic over $V$, and suppose that

$$
V[G] \Vdash \text { “ } \mathfrak{X} \text { is } \omega \text {-expandable." }
$$

Then

$$
V \vDash \text { “ } \mathfrak{X} \text { is } \theta \text {-expandable." }
$$

Proof. Without loss of generality we may assume that $X$ is infinite and $X \subset \rho$. In $V[G]$, let us define the functions $\psi_{G}: \omega \times \rho \rightarrow 2$ and $\chi_{G}: \rho \rightarrow \omega$ by putting

(a) $\psi_{G}=\bigcup G$ for $P=\operatorname{Fn}(\omega \times \rho, 2)$;

(b) $\psi_{G}(\langle k, \alpha\rangle)=i \quad(i=0,1)$ iff $\left[\left\{h \in 2^{\omega \times \rho}: h(\langle k, \alpha\rangle)=i\right\}\right]^{V} \in G$ for $P=M_{\omega \times \rho}$;

(c)

$$
\chi_{G}(\alpha)=\left\{\begin{array}{l}
0 \text { if } \psi_{G}(\langle k, \alpha\rangle)=0 \text { for every } k \in \omega, \\
\min \left\{k \in \omega: \psi_{G}(\langle k, \alpha\rangle)=1\right\} \quad \text { otherwise. }
\end{array}\right.
$$

By assumption there is an $f \in V[G]$ such that

$$
V[G] \Vdash \varphi\left(f, \chi_{G}\right),
$$

where $\varphi\left(f, \chi_{G}\right)$ stands for

" $f: X \rightarrow X$ is a function and for every $x \in X$, there is a $t \in X$ with $T\left(x, t, \chi_{G}, f\right)$ finite."

Thus there are $\dot{\mathfrak{f}} \in V^{P}, p \in G$ such that $\operatorname{val}(\dot{\mathfrak{f}}, G)=f$ and $p \Vdash \varphi\left(\dot{\mathfrak{f}}, \chi_{\check{G}}\right)$.

As in the proof of Lemma 2.7 , for each $n \geq 2$ let $\mathscr{L}_{n} \subset[P]^{<\omega}$ be an $n$ dowment below $p$. For each $z \in X$, take a maximal antichain $A(z)$ below $p$ of conditions deciding the value of $\dot{f}$ at $z$. For every $n \geq 2$ and $z \in X$, fix an $L(n, z) \in \mathscr{L}_{n}$ with $L(n, z) \subset A(Z)$. For each $n \geq 2$, take a function $g_{n}: X \rightarrow X$ in $V$ such that

$$
\begin{gathered}
\text { if } y \in \operatorname{dom}(r), q \in L(n, y), u \in X, \text { and } q \Vdash \dot{\mathfrak{f}}(y)=u, \\
\text { then } U\left(y, g_{n}(y)\right) \subset U(y, u) .
\end{gathered}
$$

We are going to show that $\left\{g_{n}: n \geq 2\right\}$ witnesses $\theta$-expandability of $\mathfrak{X}$ in $V$. To see this, let $x \in X$.

Since $p \Vdash \varphi\left(\dot{\mathfrak{f}}, \chi_{\dot{G}}\right)$, there are $t \in X, k \in \omega$, and $p(x) \leq p$ with

$$
p(x) \Vdash\left|T\left(x, t, \chi_{\check{G}}, \dot{\mathfrak{f}}\right)\right| \leq k .
$$

Let (a) $n>(p s(p(x))+1)\left(2^{(k+1)^{2}}+3\right)$.

Further, let

$$
D=\left\{\begin{array}{l}
\operatorname{dom}(p(x)) \quad \text { if } P=\operatorname{Fn}(\omega, 2) ; \\
\text { the } D \text { from Proposition } 2.6 \text { applied for } p(x) \in M_{\omega \times \rho} \\
\text { and } m=(k+1)^{2}-1 \quad \text { if } P=M_{\omega \times \rho} .
\end{array}\right.
$$


Indirectly assume that $\left|S\left(x, t, g_{n}\right)\right| \geq \omega$. Then there are $y_{0}, \ldots y_{k} \in \operatorname{dom}(r)$ such that

(i) $r\left(y_{i}\right)=a_{i}(i=0, \ldots, k)$ are pairwise distinct;

(ii) $\left(\omega \times\left\{a_{i}\right\}\right) \cap D=\varnothing \quad(i=0, \ldots, k)$;

(iii) $U(x, t) \cap U\left(y_{i}, g_{n}\left(y_{i}\right)\right) \neq \varnothing \quad(i=0, \ldots, k)$.

Let

$$
\delta_{i j}= \begin{cases}1 & \text { if } i=j \\ 0 & \text { if } i \neq j\end{cases}
$$

be Kronecker's delta, and consider

$$
p^{\prime}=\left\{\begin{array}{l}
p(x) \cup\left\{\left\langle\left\langle j, a_{i}\right\rangle, \delta_{j i}\right\rangle: i, j=0, \ldots, k\right\} \text { if } P=\operatorname{Fn}(\omega \times \rho, 2) \\
\left.p(x) \cap\left[\left\{h \in 2^{\omega \times \rho}: h\left(\left\langle j, a_{i}\right\rangle\right)=\delta_{j i} \text { for } i, j=0, \ldots, k\right)\right\}\right] \text { if } P=M_{\omega \times \rho} .
\end{array}\right.
$$

By (ii), $p^{\prime}$ is well defined and has $p(x)$-size $<2^{(k+1)^{2}}+2$ in both cases. Thus by (a), $p^{\prime}$ has $p$-size $<n$. Since $\mathscr{L}_{n}$ is an $n$-dowment, there are $q_{i} \in L\left(n, y_{i}\right)$ $(i=0, \ldots, k)$ such that $\left\{p^{\prime}, q_{0}, \ldots, q_{k}\right\}$ has a common lower bound $r$.

Since $r \leq p^{\prime} \leq p(x)$, it follows that

$$
r \Vdash\left|T\left(x, t, \chi_{\check{G}}, \dot{\mathfrak{f}}\right)\right| \leq k
$$

and

$$
r \Vdash \chi_{\check{G}}\left(a_{i}\right)=i \quad(i=0, \ldots, k) .
$$

Let $u_{i} \in X \quad(i=0, \ldots k)$ be such that $q_{i} \Vdash \dot{\mathfrak{f}}\left(y_{i}\right)=u_{i}$. Then by (iii) and $(*)$,

$$
U(x, t) \cap U\left(y_{i}, u_{i}\right) \neq \varnothing .
$$

By $(* * *),(i)$, and absoluteness,

$$
r \Vdash\left|T\left(x, t, \chi_{\check{G}}, \dot{\mathfrak{f}}\right)\right| \geq k+1
$$

in contradiction with $(* *)$.

Lemma 2.10, Definition 2.11, and Proposition 2.12 are identical to 1.10 , 1.11 , and 1.12, respectively. Lemma 2.13 is the same as Lemma 1.13, except that $\Phi(\mathfrak{X})$ now stands for " $\mathfrak{X}$ is not $\theta$-expandable." The proofs of 2.13 and 1.13 are exactly the same. We must redefine "splendid."

Definition 2.14. Let $\kappa, \nu$ be cardinals with $\omega \leq \nu<\nu^{+} \leq \operatorname{cf}(\kappa)$, let $X$ be a countably paracompact space with $|X| \geq \kappa$ and $h(x) \leq \nu, \mathscr{Y}=\left\{Y_{a}: a \in X\right\}$ be a closed discrete collection in $X, Y=\bigcup \mathscr{Y}$, and let us fix a relevant $A$ structure $\mathfrak{X}=\langle X, r, U\rangle$ for $X$ and $\mathscr{Y}$. For every subset $Z$ of $X$ and every point $x \in Z$, let

$$
C(x, Z)=\bigcap\{\overline{U(x, u)}: u \in Z\},
$$

and for every $a \in X$, let

$$
Y_{a}(Z)=\bigcup\left\{C(y, Z): y \in Y_{a} \cap Z\right\} .
$$


Then we shall say that a subset $Z \subset X$ is splendid if the following two conditions hold:

(a) $\mathscr{Y}(Z)=\left\{Y_{a}(Z): a \in X\right\}$ is a locally finite collection in $X$;

(b) for every $x \in Z, C(x, Z)$ is a compact subset of $X$ and $\{U(x, u)$ : $u \in Z\}$ is a neighborhood base for $C(x, Z)$ in $X$.

Proposition 2.15. With the definitions above, if $Z$ is a splendid subset of $X$, then the substructure $\left\langle Z, r \cap Z^{2}, U \cap Z^{3}\right\rangle$ of $\mathfrak{X}$ is filtered and $\omega$-expandable.

Proof. This follows immediately from the definition of "splendid" and the countable paracompactness of $X$.

The following "automatic character reduction" lemma was proved by the author $\left[\mathrm{B}_{2}\right]$. He does not know whether $h(X)=\omega$ in it can be weakened to $h(X)<2^{\omega}$.

Lemma $2.16\left[\mathrm{~B}_{2}\right]$. In a countably paracompact space $X$ with $h(X)=\omega$, every discrete collection $\mathscr{Y}=\left\{Y_{a}: a \in X\right\}$ has a locally finite expansion $\mathscr{R}=\left\{R_{a}: a \in X\right\}$ by $G_{\mu}$-sets, where $\mu=|\mathscr{Y}|+\omega=\left|\left\{a \in X: Y_{a} \neq \varnothing\right\}\right|+\omega$.

Proposition 2.17 is identical to 1.17 .

Lemma 2.20. Under the assumptions of Definition 2.14, if $\nu=\omega$, then

$$
\Gamma=\left\{Z \in[X]^{<\kappa}: Z \text { is splendid }\right\} \text { is } a\left(\kappa, \omega_{1}\right) \text {-c.u.b. on }[X]^{<\kappa} \text {. }
$$

The proof is similar to the proof of Lemmas 1.18-1.20 (actually, it is slightly simpler because we can forget about the $G(a)$ 's) and is therefore omitted.

Finally, the above sequence of lemmas can be put together to a proof of Theorem 2.1 in the same way the corresponding lemmas were put together in the first section to yield a proof of Theorem 1.1 .

Final remarks. (1) The author does not know whether $h(X)=\omega$ can be improved to $h(X)<2^{\omega}$ in Theorem 2.1. Lemma 2.16 is the only place in the proof of Theorem 2.1 where $h(X)=\omega$ is made use of, so it would be enough to improve or bypass this lemma.

(2) By Fleissner's famous result $\left[\mathrm{F}_{3}\right]$, if all normal Moore spaces are metrizable, then there is an inner model with a measurable cardinal. Therefore to prove the consistency of either "every normal space $X$ with $h(X)=\omega$ is collectionwise normal" or "every countably paracompact space $X$ with $h(X)=\omega$ is expandable," some large cardinal hypotheses are necessary, since both of these statements imply that normal Moore spaces are metrizable.

It is not known, however, what happens, if " $h(X)=\omega$ " is replaced by "locally compact." The following problem is probably the most important question left open in this paper.

Problem. Can the consistency of either "normal, locally compact spaces are collectionwise normal" or "countably paracompact, locally compact spaces are expandable" be proved without large cardinals? 
Acknowledgments. The research incorporated in this paper was completed while the author was attending a set theory conference, STACY, August 1987, Toronto. Discussions with the conference participants, in particular with F. Tall, B. Beaudoin, W. Fleissner, P. Nyikos, and W. Zwicker, were very helpful. I would also like to thank the organizing committee for financial assistance.

\section{REFERENCES}

$\left[\mathrm{A}_{1}\right]$ A. V. Arhangel'skii, The Suslin number and cardinality. Characters of points in sequential bicompacta, Dokl. Akad. Nauk SSSR 192 (1970), 597-601.

$\left[\mathrm{A}_{2}\right]$ - The property of paracompactness in the class of perfectly normal, locally bicompact spaces, Dokl. Akad. Nauk SSSR 203 (1972), 517-520.

[AZ] K. Alster and P. Zenor, On the collectionwise normality of generalized manifolds, Topology Proc. 1 (1976), 125-127.

$\left[\mathrm{B}_{1}\right] \quad$ Z. Balogh, Paracompactness in locally Lindelöf spaces, Canad. J. Math. 38 (1986), 719-727.

$\left[\mathrm{B}_{2}\right] \ldots$ Locally compact, countably paracompact spaces in the constructible universe, Topology Appl. 29 (1988), 19-26.

[Bu] D. K. Burke, PMEA and first countable, countably paracompact spaces, Proc. Amer. Math. Soc. 92 (1984), 455-460.

[CZ] J. Chaber and P. Zenor, On perfect subparacompactness and a metrization theorem for Moore spaces, Topology Proc. 2 (1977), 401-407.

$\left[\mathrm{D}_{1}\right]$ P. Daniels, Normal, locally compact, boundedly metacompact spaces are paracompact: an application of Pixley-Roy spaces, Canad. J. Math. 35 (1983), 807-823.

$\left[\mathrm{D}_{2}\right]$ -, On collectionwise Hausdorffness in countably paracompact, locally compact spaces, preprint.

$\left[\mathrm{D}_{3}\right]$ - When countably paracompact, locally compact, screenable spaces are paracompact, Topology Appl. 28 (1988), 113-125.

[DG] P. Daniels and G. Gruenhage, A perfectly normal, locally compact, non-collectionwise normal space from $\diamond^{*}$, Proc. Amer. Math. Soc. 95 (1985), 115-118.

[Do ${ }_{1}$ A. Dow, Remote points in large products, Topology Appl. 16 (1983), 11-17.

$\left[\mathrm{Do}_{2}\right]$ _ Some linked subsets of posets, Israel J. Math. 59 (1987), 353-376.

[DS] K. Devlin and S. Shelah, A note on the normal Moore space conjecture, Canad. J. Math. 31 (1979), 241-251.

[DTW] A. Dow, F. Tall, and W. Weiss, New proofs of the consistency of the normal Moore space conjecture (revised), preprint.

$\left[\mathrm{F}_{1}\right] \quad$ W. G. Fleissner, Normal Moore spaces in the constructible universe, Proc. Amer. Math. Soc. 46 (1974), 294-298.

$\left[\mathrm{F}_{2}\right]$ - When is Jones' space normal', Proc. Amer. Math. Soc. 50 (1975), 375-378.

$\left[\mathrm{F}_{3}\right] \quad$ _. If all normal Moore spaces are metrizabie, then there is an inner model with a measurable cardinal, Trans. Amer. Math. Soc. 273 (1982), 365-373.

$\left[\mathrm{F}_{4}\right] \ldots$ - Discrete sets of singular cardinality, Proc. Amer. Math. Soc. 88 (1983), 743-745.

$\left[\mathrm{F}_{5}\right] \quad$, The normal Moore space conjecture and large cardinals, Handbook of Set-Theoretic Topology, North-Holland, 1984, pp. 733-760.

$\left[\mathrm{F}_{6}\right] \ldots$ - Homomorphism axioms and lynxes, Contemp. Math. 31 (1984), 79-97.

$\left[\mathrm{F}_{7}\right] \ldots$ Burke's theorem from the product category extension axiom, Topology Proc. 10 (1985), $55-57$.

$\left[\mathrm{F}_{8}\right] \quad-$ Left-separated spaces with point-countable hases, Trans. Amer. Math. Soc. 294 (1986), 665-674.

[Fr] D. Fremlin, handwritten notes. 
$\left[\mathrm{G}_{1}\right] \quad$ G. Gruenhage, Paracompactness in normal, locally connected, locally compact spaces, Topology Proc. 4 (1979), 393-405.

$\left[\mathrm{G}_{2}\right] \quad$, Two normal locally compact spaces under Martin's axiom, Topology Proc. 9 (1984), 297-305.

$\left[\mathrm{K}_{1}\right] \quad \mathrm{K}$. Kunen, Set theory (an introduction to independence proofs), North-Holland, 1980.

$\left[\mathrm{K}_{2}\right] \_$, Random and Cohen reals, Handbook of Set-Theoretic Topology, North-Holland, 1984, pp. 887-911.

[KM] A. Kanamori and M. Magidor, The evolution of large cardinal axioms in set theory, Proc. Conf. on Higher Set Theory, Lecture Notes in Math., vol. 669, Springer-Verlag, Berlin, 1978, pp. 99-275.

[KV] K. Kunen and J. E. Vaughan, editors, Handbook of set-theoretic topology, North-Holland, 1984.

[L] D. J. Lane, Paracompactness in perfectly normal, locally connected, locally compact spaces, Proc. Amer. Math. Soc. 80 (1980), 693-696.

$\left[\mathrm{N}_{1}\right]$ P. Nyikos, A provisional solution to the normal Moore space problem, Proc. Amer. Math. Soc. 78 (1980), 429-435.

$\left[\mathrm{N}_{2}\right]$ _. The normal Moore space problem, Topology Proc. 3 (1978), 473-493.

[R] M. E. Rudin, The undecidability of the existence of a perfectly normal manifold, Houston J. Math. 5 (1979), 249-252.

[RZ] G. M. Reed and P. Zenor, Metrization of Moore spaces and generalized manifolds, Fund. Math. 91 (1976), 203-210.

[S] S. Shelah, Remarks on $\lambda$-collectionwise Hausdorff spaces, Topology Proc. 2 (1977), 583-592.

$\left[\mathrm{T}_{1}\right] \quad \mathrm{F}$. D. Tall, Set-theoretic consistency results and topological theorems concerning the normal Moore space conjecture and related problems, Thesis, 1969, Dissertationes Math. 148 (1977), $1-53$.

$\left[\mathrm{T}_{2}\right]-$, Collectionwise normality without large cardinals, Proc. Amcr. Math. Soc. 85 (1982), $100-102$.

$\left[\mathrm{T}_{3}\right]$ _. Normality versus collectionwise normality, Handbook of Set-Theoretic Topology, North-Holland, 1984, pp. 685-732.

$\left[\mathrm{T}_{4}\right] \quad$ Countably paracompact Moore spaces are metrizable in the Cohen model, Topology Proc. 9 (1984), 145-148.

$\left[\mathrm{T}_{5}\right] \ldots$ - Corrigendum to "Countably paracompact Moore spaces are metrizable in the Cohen model", Topology Proc. 10 (1985), 175-176.

$\left[\mathrm{T}_{6}\right]-$, Covering and separation properties in the Easton model, Topology Appl. 28 (1988), $155-164$.

$\left[\mathrm{T}_{7}\right] \quad$ A note on collectionwise normality of locally compact normal spaces, Topology Appl. 28 (1988), 165-170.

$\left[\mathrm{T}_{8}\right] \quad$ - Are locally compact normal spaces collectionwise normal?, Questions and Answers in Topology 2 (1984), 77-82.

$\left[\mathrm{T}_{9}\right] \quad$ - On the collectionwise normality of locally compact normal spaces, preprint.

[Sm] J. C. Smith, On $\theta$-expandable spaces, Glasnik Mat. 11 (31) (1976), 335-346.

[SmK] J. C. Smith and L. Krajewski, Expandability and collectionwise normality, Trans. Amer. Math. Soc. 160 (1971), 437-451.

$\left[\mathrm{W}_{1}\right]$ S. Watson, Locally compact normal spaces in the constructible universe, Canad. J. Math. 34 (1982), 1091-1096.

$\left[\mathrm{W}_{2}\right]-$ Separation in countably paracompact spaces. Trans. Amer. Math. Soc. 290 (1985), $831-842$.

$\left[\mathrm{W}_{3}\right] \ldots$ Locally compact normal metalindelöf spaces may not be paracompact: an application of uniformization and Suslin lines, Proc. Amer. Math. Soc. 98 (1986), 676-680.

Department of Mathematics, Miami University, Oxford, Ohio 45056 